\title{
Sindrome de Brash: bradicardia, falla renal, bloqueo auriculoventricular, shock e hiperkalemia. Reporte de un caso.
}

\section{Of Brash syndrome: bradycardia syndrome, kidney failure, atrioventricular block, shock, and hyperkalemia. A case report.}

MD. Nelson Chacón Toba. ${ }^{1}$, MD. Adriana Calderón Peñaherrera. ${ }^{2} \&$ MD Carlos Haro Erazo. ${ }^{3}$

\begin{abstract}
.
DOI: https://doi.org/10.33262/cienciadigital.v2i2.120

The Brash syndrome, term proposed by Dr. Josh Farkas Assistant Professor of Pulmonology and Critical Care at the University of Vermont / Burlington Vermont / USA. UU It is a pathological entity that is due to a chain of pathophysiological events that usually occurs in elderly patients with underlying heart disease that is managed with a beta-blocker or calcium channel blockers. Basal renal dysfunction is often involved. This combination of events plus hyperkalemia potentiate the adverse effects of AV nodal blockers to cause bradycardia, hypoperfusion and shock. Hypoperfusion in turn causes a worsening of kidney failure and in this way a vicious circle is created that ends in catastrophic consequences. The Brash syndrome has been described by several authors in case studies, however, this entity continues to be poorly recognized because generally the entire symptomatology is completely attributed to hyperkalemia, meanwhile, the presence of AV nodal blockers in the background may seem less important, however, one of the characteristics of this syndrome is the presence of moderately elevated potassium values and an unexplained severe cardiac symptomatology, here lies the importance of early recognition and appropriate treatment. The pathophysiological key of Brash syndrome is the ability of hyperkalemia to potentiate the action of AV nodal blockers and in this way produce symptomatic bradycardia and shock.
\end{abstract}

\footnotetext{
${ }^{1}$ Hospital de la Policía Nacional Quito, Quito, Ecuador, nelmarcect@ gmail.com ${ }^{2}$ Hospital de la Policía Nacional Quito, Quito, Ecuador, nelmarcect@gmail.com ${ }^{3}$ Sociedad de Lucha contra en Cáncer SOLCA Núcleo Chimborazo, Ecuador, carlos.chex87@ gmail.com
} 
Keywords: Symptomatic Bradycardia, Renal Failure, Calcium Channel Blockers, Beta Blockers, Shock and Hyperkalemia.

\section{Resumen.}

El síndrome de Brash, término propuesto por el Dr. Josh Farkas Profesor Asistente de Neumología y Cuidados Críticos en la Universidad de Vermont/Burlington Vermont/EE. UU. Es una entidad patológica que se debe a una cadena de eventos fisiopatológicos que se presenta generalmente en pacientes ancianos con enfermedad cardíaca subyacente que se manejan con betabloqueantes o bloqueadores de canales de calcio. La disfunción renal basal a menudo está involucrada. Esta combinación de eventos más la hipercalemia, potencian los efectos adversos de los bloqueadores del nódulo AV para causar bradicardia, hipoperfusión y shock. La hipoperfusión a su vez causa un empeoramiento de la insuficiencia renal y de esta manera se crea un círculo vicioso que termina en catastróficas consecuencias. El síndrome de Brash ha sido descrito por varios autores en estudio de casos, sin embargo, esta entidad sigue siendo poco reconocida debido que generalmente toda la sintomatología se atribuye completamente a la hipercalemia, mientras tanto, la presencia de bloqueadores de nódulos AV en el fondo puede parecer menos importante, sin embargo, una de las caracteristicas de este síndrome es la presencia de valores moderadamente elevados de potasio y una inexplicable sintomatología cardiaca severa, aquí radica la importancia de su reconocimiento precoz y tratamiento adecuado. La clave fisiopatológica del síndrome Brash es la capacidad de la hipercalemia para potenciar la acción de los bloqueadores del nódulo AV y de esta manera producir bradicardias sintomáticas y shock.

Palabras Claves: Bradicardia Sintomática, Insuficiencia Renal, Bloqueadores de los Canales de Calcio, Beta Bloqueadores, Shock E Hipercalemia.

\section{Introducción .}

El síndrome Brash representa una superposición de eventos entre la hipercalemia y la intoxicación por bloqueador del nodo aurículo ventricular (AV) en el contexto de un paciente con falla renal aguda o crónica. Se presenta generalmente en pacientes ancianos con enfermedad cardíaca subyacente que se maneja con un betabloqueante o calcio antagonistas. [1]

La hipercalemia es un problema metabólico relativamente común en pacientes críticamente enfermos, potencialmente grave y que puede inducir arritmias cardíacas mortales cuando se encuentra en valores superiores a los $7 \mathrm{mEq} / \mathrm{L}$. Acker et al informaron que el $75 \%$ de los 
pacientes con hipercalemia severa tenían insuficiencia renal como causa base y el $67 \%$ tenían un fármaco predisponente. [2,3]

En el paciente con enfermedad renal crónica puede presentar hipercalemia debido a la retención de este electrolito, esto a su vez provoca alteraciones de la conductibilidad a nivel cardiaco, generando alteraciones del ritmo cardíaco tales como bloqueos, bradicardia o latidos de escape, si el efecto a nivel cardiaco es sostenido se puede dar lugar al daño miocárdico irreversible. [4,5]

Los bloqueadores beta (BB), y bloqueadores de los canales de calcio (CCB) son medicamentos comúnmente utilizados en pacientes hipertensos, los mismos que deben ser evaluados por un especialista debido al riesgo de efectos adversos en casos de intoxicación por los mismos; estos medicamentos son principalmente inotrópicos negativos y tienen efectos sobre el corazón que pueden conducir a una bradicardia profunda e hipotensión debido a una sobredosis. [6]

El síndrome de Brash a menudo se atribuye completamente a la hipercalemia, mientras que la presencia de bloqueadores de nódulos AV parece menos importante, sin embargo, los pacientes con síndrome de BRASH pueden tener sintomatología severa con una hipercalemia leve debido a la sinergia entre la hipercalemia y el bloqueador del nódulo AV. [1]

\section{Información del paciente .}

Paciente femenina adulta mayor de 72 años, nacida y residente en Quito-Pichincha, instrucción primaria completa, casada, ama de casa, etnia mestiza, religión católica, grupo sanguíneo ORH+.

\section{Antecedentes clínicos:}

1. Diabetes Mellitus tipo 2 diagnosticada hace 24 años en tratamiento con insulina de acción lenta 26 UI en la mañana,

2. Hipertensión Arterial hace 1 año en tratamiento con diltiazem $120 \mathrm{mg}$ vía oral cada 12 horas, amlodipino $10 \mathrm{mg}$ vía oral una vez al día y doxasocina $4 \mathrm{mg}$ vía oral una vez al día,

3. Enfermedad Renal Crónica estadio 4 hace 1 año en tratamiento con furosemida $40 \mathrm{mg}$ vía oral cada 12 horas, simvastatina $20 \mathrm{mg}$ vía oral una vez al día y complejo B,

4. Hipotiroidismo diagnosticado hace 15 años en tratamiento con levotiroxina 50ug vía oral una vez al día.

\section{Hábitos:}

- Alimentarios: 3-5 veces al día

- Miccional: 4-5 veces al día

- Defecatorio: 1-2 veces al día 
- Tabaquismo: no refiere

- Alcoholismo: no refiere

- Drogas: no refiere

- Alergias: no refiere

- Transfusiones: no refiere

Paciente ingresa con cuadro clínico compatible con una infección de vías urinarias de 48 horas de evolución, con los siguientes signos vitales: TA: 170/70 mmhg, FC: 80 lpm, FR: 20 rpm, Temperatura: $39^{\circ}$ y una escala de coma de Glasgow: 15/15.

\section{Hallazgos clínicos:}

Neurológicamente estable con una escala de coma de Glasgow de 15/15.

Sistema cardiovascular sin alteraciones evidentes, se ausculta un soplo sistólico en foco aórtico.

Tórax y pulmones sin alteraciones.

En su examen físico demuestra dolor abdominal difuso de poca intensidad, sin signos de irritación peritoneal y sin signos apendiculares.

\section{Caso clínico cronología.}

Paciente permanece 24 horas en hospitalización en regular estado general, hemodinámicamente estable, y sin complicaciones. Se le administra antibiótico-terapia, insulina por escapes, diurético de ASA, antihipertensivos (amlodipino 10mg QD y diltiazem $120 \mathrm{mg}$ BID), sin embargo 12 horas posteriores paciente en malas condiciones generales, con bradicardia (Tabla1), deterioro del estado de conciencia, diaforética e hipotensa.

Signos Vitales: TA:80/60 mmHg FC: 36 lmp, SATO2: 78\% y FIO2 21\%.

Tabla1: Signos Vitales

\begin{tabular}{|c|c|c|c|c|c|c|}
\hline \\
\hline \multicolumn{7}{|c|}{\begin{tabular}{l}
\multicolumn{2}{l}{ SIGNOS VITALES } \\
FECHA HORA
\end{tabular}} \\
\hline $16 / 09 / 17$ & $06 \mathrm{AM}$ & 38 & $80 \mathrm{lpm}$ & $22 \mathrm{rpm}$ & $153 / 94 \mathrm{mmHg}$ & $90 \%$ \\
\hline $16 / 09 / 17$ & $14 \mathrm{PM}$ & 36,9 & $71 \mathrm{lpm}$ & $20 \mathrm{rpm}$ & $140 / 80 \mathrm{mmHg}$ & $88 \%$ \\
\hline $16 / 09 / 17$ & $22 \mathrm{PM}$ & 36,2 & $60 \mathrm{lpm}$ & $20 \mathrm{rpm}$ & $90 / 45 \mathrm{mmHg}$ & $82 \%$ \\
\hline 17/09/17 & $04 \mathrm{AM}$ & 36 & $66 \mathrm{lpm}$ & $20 \mathrm{rpm}$ & $90 / 65 \mathrm{mmHg}$ & $96 \%$ (2lts 02$)$ \\
\hline 17/09/17 & $06 \mathrm{AM}$ & 36,2 & $60 \mathrm{lpm}$ & $18 \mathrm{rpm}$ & $90 / 60 \mathrm{mmHg}$ & $94 \%$ (2lts 02 ) \\
\hline 17/09/17 & $08 \mathrm{AM}$ & 36 & $32 \mathrm{lpm}$ & $24 \mathrm{rpm}$ & $80 / 60 \mathrm{mmHg}$ & $85 \%$ (3lts 02 ) \\
\hline
\end{tabular}

NOTA: Signos Vitales reportados en el Servicio de Medicina Interna del Hospital Quito №1 de la Policíaa Nacional. 


\section{Evaluación diagnóstica:}

\section{Laboratorio}

Se evidencia en sus controles gasométricos una acidosis metabólica severa parcialmente compensada e hipoxemia moderada, mismos parámetros que mejoran con el transcurso de los dias (Tabla 2).

En sus resultados de biometría y química sanguínea podemos evidenciar una leucocitosis con desviación a la izquierda, sugestiva de un proceso infeccioso, además, se nota una función renal comprometida con una creatinina sobre los $3 \mathrm{md} / \mathrm{dL}$ y un clearance de creatinina de 15 con lo cual es catalogada como como una Falla Renal Crónica estadio 4. (Tabla3) Se evidencia unos valores de glicemia sobre los $200 \mathrm{mg} / \mathrm{dL}$, con una hemoglobina glicosilada > $11 \mathrm{mg} / \mathrm{dL}$ que sugiere una diabetes mal controlada, finalmente se nota una hipercalemia moderada con un valor inicial de 6,4 mEqv/L. (Tabla3)

Tabla2: Gasometrías

\begin{tabular}{|l|l|l|l|l|l|l|c|}
\hline \multicolumn{5}{|c|}{$17 / 09 / 2017$} & \multicolumn{1}{l}{$18 / 09 / 2017$} & $19 / 09 / 17$ \\
\hline HORA & $\mathbf{0 7 : 4 8}$ & $\mathbf{1 3 : 2 8}$ & $\mathbf{1 6 : 4 8}$ & $\mathbf{2 0 : 5 1}$ & $\mathbf{0 6 : 0 0}$ & $\mathbf{2 1 : 5 1}$ & $\mathbf{1 3 : 2 8}$ \\
\hline Ph & 7.26 & 7.18 & 7.20 & 7.33 & 7.49 & 7.42 & 7.41 \\
\hline PaC02 & 23.4 & 20.9 & 24.8 & 26.2 & 22.7 & 26.9 & 31.8 \\
\hline Pa02 & 48.6 & 100 & 59.1 & 55.3 & 78.7 & 59.3 & 47.0 \\
\hline HC03 & 10.6 & 7.9 & 9.6 & 13.9 & 17.1 & 17.6 & 20.3 \\
\hline EB & -14.3 & -18.3 & -16.6 & -10.0 & -4.0 & -5.1 & -3.2 \\
\hline Sa02 & 76.7 & 95.3 & 82.8 & 86.3 & 96.5 & $91.4 \%$ & $83.2 \%$ \\
\hline HCT & 22.1 & 32.1 & 31.5 & 29.4 & 33.4 & $34.1 \%$ & $32.8 \%$ \\
\hline TCO2 & 11.3 & 8.5 & 10.4 & 14.7 & 17.8 & 18.4 & 21.2 \\
\hline
\end{tabular}

NOTA: Gasometrías Arteriales reportadas en el Servicio de Terapia Intensiva del Hospital Quito №1 de la Policía Nacional.

Tabla3: Laboratorios

\begin{tabular}{|lccc|}
\hline \multicolumn{4}{c|}{ EXAMENES LABORATORIO } \\
\hline & $17 / 09 / 2017$ & $18 / 09 / 2017$ & $19 / 09 / 2017$ \\
\hline LEUCOCITOS & 12.090 & 15.430 & 12.380 \\
\hline NEUTROFILOS & $86.1 \%$ & $78 \%$ & $75 \%$ \\
\hline LINFOCITOS & $7 \%$ & $13.8 \%$ & $13.6 \%$ \\
\hline HEMOGLOBINA & 10.1 & 9.5 & 10.1 \\
\hline HEMATOCRITO & $29.9 \%$ & $28.9 \%$ & 30.3 \\
\hline CREATININA & 3.25 & 3.47 & 3.66 \\
\hline BUN & 36.9 & 42.3 & 37.3 \\
\hline GLUCOSA & 350 & 229 & 145 \\
\hline POTASIO & 6.4 & 4.4 & 4.8 \\
\hline SODIO & 128 & 134 & 133 \\
\hline CLORO & 102 & 102 & 103 \\
\hline HBA1C & 11.6 & & \\
\hline
\end{tabular}

NOTA:Laboratorios reportados en el Servicio de Terapia Intensiva del Hospital Quito №1 de la Policía Nacional. 


\section{Electrocardiograma}

Su electrocardiograma muestra ritmo nodal o de la unión, bradicardia con fc de $40 \mathrm{lpm}$, ausencia de ondas P y un intervalo QTe de $425 \mathrm{mseg}$ sin alteraciones de la onda $\mathrm{T}$ y no se evidencian signos de isquemia o necrosis miocárdica (Figura1).

Figura 1: Electrocardiograma

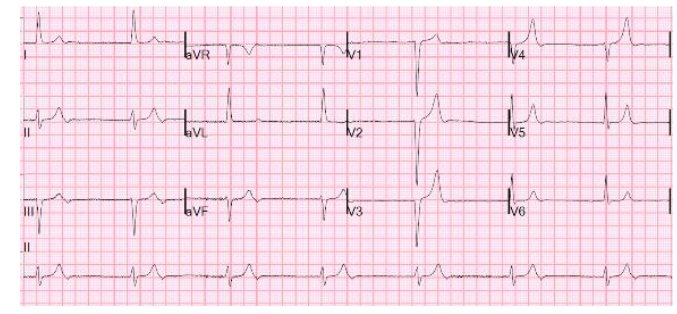

NOTA: Electrocardiograma en donde se evidencia un ritmo de la unión, con frecuencias cardiacas en $40 \mathrm{lpm}$, sin presencia de ondas $\mathrm{P}$ y sin alteraciones en la onda $\mathrm{T}$.

\section{Diagnósticos .}

Paciente ingresa con los siguientes diagnósticos:

1. Bradicardia Extrema.

2. Intoxicación por Bloqueadores de Canales de Calcio.

3. Hipercalemia moderada.

4. Infección de Vías Urinarias Complicada.

5. Diabetes Mellitus Tipo 2 Descompensada.

6. Enfermedad Renal Crónica Estadio 4 Reagudizada

\section{Intervención terapeutica .}

La paciente es trasladada a la Unidad de Terapia Intensiva para el manejo inicial de una bradicardia extrema con frecuencias cardiacas que llegan a 36 latidos por minuto, con hipotensión arterial, signos de shock y deterioro del estado de conciencia:

1. Solución Salina 0,9\% IV en bolo de 500 centímetros cúbicos en ese momento.

2. Nebulizaciones con Salbutamol 20 miligramos en ese momento y cada 4 Horas.

3. Gluconato de Calcio 2 gramos intravenosos en ese momento y cada 6 horas.

4. Bicarbonato 10 Ampollas ( 89 mili equivalentes) intravenoso en ese momento.

5. Suspender Bloqueadores de canales de Calcio.

6. Insulina Cristalina 10 unidades internacionales más 100 centímetros cúbicos de dextrosa al 50\% intravenosos en una hora. 
Posterior a su estabilización hemodinámica

\section{Furosemida IV.}

Revierte cuadro parcialmente con frecuencias cardiacas de hasta 90 lpm, sim embargo tiende a la bradicardia por segunda ocasión con FC de 50 lpm (posterior a la administración de

\section{Diltiazem)}

1. Atropina $0,5 \mathrm{mg}$ IV en ese momento.

2. Nebulizaciones con Salbutamol.

3. Gluconato De Calcio 2 Gr IV en ese momento.

Algunas horas posteriores a segundo evento paciente hemodinámicamente estable sin signos de bajo gasto, frecuencias cardiacas de 75 a 80 lpm, sin nuevas complicaciones.

Aritmología recomienda suspender definitivamente bloqueadores de canales de calcio.

\section{Discusión .}

El síndrome BRASH representa un cuadro clínico de compleja identificación y que puede desencadenarse por múltiples causa ya sean de origen infeccioso, metabólico o medicamentoso, además esta entidad hace referencia a una superposición entre hipercalemia e intoxicación por bloqueadores del nódulo AV, lo cual permite que con dosis adecuadas de estos medicamentos o con hipercalemia leves se desarrolle sintomatología severa (Ejemplos: episodios de bradicardia extrema, hipoperfusión y shock).

El Síndrome de BRASH se presenta como el punto intermedio entre la intoxicación por BB o CCB y la hipercalemia. [1]

El tratamiento del síndrome Brash se centra en la terapia dirigida a la corrección de la hipercalemia y posible envenenamiento por medicamentos bloqueadores del nódulo AV, catecolaminas para apoyar su perfusión e incluso el uso de marcapasos para la bradicardia extrema.

Uno de las principales conclusiones de este apartado es recalcar que el algoritmo de bradicardia ACLS no funciona bien en el síndrome BRASH debido a que los factores desencadenantes no siempre responden adecuadamente a la administración de atropina o a la estimulación transcutanea. Se debe enfatizar además el uso de Gluconato de Calcio intravenoso para una adecuada reversión del cuadro clínico.

Es primordial resaltar que seguir ciegamente el algoritmo ACLS puede conducir a una sobreutilización de la estimulación transvenosa y una mayor posibilidad de fracaso terapéutico en este tipo de pacientes, motivo por el cual es recomendable individualizar cada caso en particular y fortalecer los conocimientos sobre las interacciones medicamentosas y sus variaciones en los pacientes con falla renal. 
La hipercalemia es un factor de riesgo independiente para la mortalidad en pacientes con enfermedad cardiovascular, enfermedad renal crónica (ERC) en etapa terminal. [7]

Este artículo también intenta resaltar algunas de las características que nos pueden ayudar a distinguir un síndrome de Brash de otras entidades.

Los hallazgos de EKG en la hipercalemia pueden ser variables, [1] mientras que la hipotensión, la supresión del nodo sinusal, los ritmos de la unión y las arritmias a menudo se producen con sobredosis del bloqueador de los canales de calcio, no obstante, la bradicardia sin otras características de hipercalemia (por ejemplo, el aumento de QRS, ondas T en pico) puede obedecer a una intoxicación por bloqueadores del nódulo AV y favorecer el diagnóstico de síndrome BRASH. [14]

\section{Tratamiento .}

Las medidas terapéuticas primordiales para el manejo del Síndrome de BRASH están orientadas principalmente a la corrección de la hipercalemia y de la bradicardia ya sea por la misma hipercalemia o por la intoxicación de medicamentos bloqueadores del nódulo AV, de esta forma se limita los efectos nocivos de la hipoperfusión y se evita que la bradicardia llegue a causar shock. [8]

La hipotensión, la bradicardia, la disminución de la resistencia vascular sistémica y el shock cardiogénico son características del envenenamiento por betabloqueante y los bloqueadores de los canales de calcio.

El tratamiento inicial es principalmente de apoyo e incluye la reanimación con solución salina, que es esencial para corregir la vasodilatación y bajas presiones de llenado cardíaco. Las terapias convencionales como la atropina, el glucagón y el calcio a menudo no mejoran el estado hemodinámico en pacientes gravemente envenenados. Las catecolaminas pueden aumentar la presión arterial y la frecuencia cardíaca, pero también aumentan las resistencias periféricas, lo que puede ocasionar una disminución del gasto cardíaco y la perfusión de los lechos vasculares. Los estimuladores cardiacos (marcapasos) son utilizados en casos de bradicardias que no mejoren a pesar de un tratamiento farmacológico adecuadamente instaurado.

El aumento de la demanda de oxígeno del miocardio que resulta de las catecolaminas y vasopresores puede ser perjudicial en el contexto de la hipotensión y la disminución de la perfusión coronaria. [16]

\section{Gluconato de calcio.}


El primer paso es administrar calcio intravenoso (IV) para mejorar la toxicidad cardíaca, si está presente. Infundir cloruro de calcio o gluconato de calcio $(10 \mathrm{ml}$ de una solución al $10 \%$ durante 2-3 minutos) [15]

\section{Insulina.}

Las infusiones intravenosas de glucosa e insulina son muy efectivas para mejorar la captación de potasio. Un régimen típico es $10 \mathrm{U}$ de insulina regular y $50 \mathrm{ml}$ de dextrosa $50 \%$ en agua. El inicio de acción es dentro de 20-30 minutos, y la duración es variable, oscilando entre 2 y 6 horas.

La insulina intravenosa (incluso cuando se administra con dextrosa) puede causar hipoglucemia. Los pacientes con daño renal agudo y enfermedad renal crónica son especialmente susceptibles

\section{Bicarbonato.}

Corregir la acidosis metabólica con bicarbonato de sodio. Debido al efecto variable de las diferentes formas de acidosis metabólica sobre el nivel sérico de potasio, esta modalidad terapéutica es menos efectiva y menos predecible para producir una respuesta hipopotasémica, especialmente en pacientes con insuficiencia renal crónica. No obstante, si la acidosis es grave, se justifica una prueba de terapia con bicarbonato de sodio parenteral. [15]

\section{Diureticos.}

La excreción renal se potencia fácilmente en pacientes con función renal normal mediante la administración de solución salina IV acompañada de un diurético de asa (p. Ej., Furosemida). [15]

\section{Catecolaminas.}

Estos medicamentos son fundamentales para el manejo de la bradicardia como medida inicial debido a que mejoran la perfusión y frecuencia cardíaca, se evita el shock y se detiene la progresión de la falla renal, sin embargo, en algunos pacientes se puede presentar un cuadro de shock refractario que requiera la administración de más de un inotrópico por lo que es importante la monitorización continua y el manejo en UCI de estos pacientes. [11]

\section{Conclusiones}

- La biomasa de los desechos orgánicos obtenidos de la granja avícola "Cynthia Elizabeth" constituyen una fuente viable para la producción de biogás tomando en consideración que, por su fácil biodegradabilidad y características, favorecen notablemente el proceso de digestión anaerobia. 
- Se define que la biomasa de la granja "Cynthia Elizabeth en las proporciones adecuadas logradas en el diseño experimental se obtiene una producción de gas metano viable para el aprovechamiento y utilización de su potencial energético.

- Con el diseño experimental implementado se logró verificar el potencial energético de la biomasa según su volumen y la cantidad de biogás producido en la degradación de la misma, obteniendo con los cálculos respectivos un volumen de $40 \mathrm{~m}^{3}$ de biomasa se logró determinar la cantidad de producción en $0,61 \mathrm{~m}^{3}$ de biogás para ser aprovechado su potencial energético, en base a este valor se pudo plantear una propuesta sostenible que pueden ser aplicadas para darle un uso a este importante recurso que no está siendo aprovechado.

- Las experiencias realizadas a nivel experimental bajo condiciones controladas, permitieron conocer el potencial máximo de producción de biogás permitiendo determinar los parámetros y condiciones de operación para la obtención de datos que permitan realizar el diseño de un biodigestor y aprovechar el potencial energético de los residuos orgánicos en la granja "Cynthia Elizabeth".

\section{Referencias bibliográficas.}

1. Josh Farkas. BRASH syndrome: Bradycardia, Renal failure, Av blocker, Shock, Hyperkalemia. PulmCrit. February 15, 2016

2. Almukdad H. Hyperkalemia Revisited. Saudi J Kidney Dis Transpl 2007;18:577-84

3. Libro electrolitos

4. Marcos Aurélio Barboza de Oliveira, MD, MsC; Antônio Carlos Brandi, MD; Carlos Alberto dos Santos, MD; Paulo Henrique Husseini Botelho, MD; José Luis Lasso Cortez, MD; Domingo Marcolino Braile, MsC, PhD. Modes of induced cardiac arrest: hyperkalemia and hypocalcemia - Literature review. Rev Bras Cir Cardiovasc 2014;29(3):432-6

5. Martín Angulo, Pedro Grille, Henry Albornoz,José Ignacio Álvez, Homero Bagnulo. Intoxicación grave por bloqueadores de los canales de calcio. Rev Méd Urug 2012; 28(3): $225-231$

6. Christopher R. H. Newton, M.D., Joao H. Delgado, M.D., and Hernan F. Gomez, M.D. Calcium and Beta Receptor Antagonist Overdose: A Review and Update of Pharmacological Principles and Management. Seminars in Respiratory and Critical Care Medicine, volume 23, number 1, 2002.

7. Fordjour, K.N., Walton, T., and Doran, J.J. Management of hyperkalemia in hospitalized patients. Am J Med Sci. 2014; 347: 93-100 
8. Angulo $\mathrm{M}$ et al. Intoxicación grave por bloqueadores de los canales de calcio. Rev Méd Urug 2012; 28(3): 225-231

9. Hou Tee Lu. Beta-blocker use and risk of symptomatic bradyarrhythmias: a hospitalbased case-control study. Journal of Geriatric Cardiology (2016) 13: 749-759

10. M. O. Hegazi* MRCP, G. Aldabie* BMBCh, S. Al-Mutairi* FRCPC and A. El Sayed MRCP. Junctional bradycardia with verapamil in renal failure - care required even with mild hyperkalaemia. Case Report. Journal of Clinical Pharmacy and Therapeutics, 2012. doi: 10.1111/j.1365-2710.2012.01352.

11. Christopher R. H. Newton, M.D.,1 Joao H. Delgado, M.D.,2 and Hernan F. Gomez, M.D. Calcium and Beta Receptor Antagonist Overdose: A Review and Update of Pharmacological Principles and Management. Seminars in respiratory and critical care medicine/volume 23, number 12002

12. Emad F. Aziz, Fahad Javed, Aleksandr Korniyenko, Balaji Pratap, Juan Pablo Cordova, Carlos L. Alviar, Eyal Herzog. Mild hyperkalemia and low eGFR a tedious recipe for cardiac disaster in the elderly: an unusual reversible cause of syncope and heart block. Heart International 2011; volume 6:e12

13. Manejo Agudo de los Trastornos Electrolíticos y del Equilibrio Ácido-Base $2^{\mathrm{a}}$ edición coordinador de la obra m.a. de la cal Ramírez.

14. MD, FACEP Edward A Ramoska'Correspondence information about the author MD, FACEP Edward A Ramoska, RN, MS Henry A Spiller, PhD Marc Winter, RPh Doug Borys , A one-year evaluation of calcium channel blocker overdoses: Toxicity and treatment-Annals of emergenci medicine.

15. Eleanor Lederer, MD, FASN Professor of Medicine, Chief, Nephrology Division, Director, Nephrology Training Program, Director, Metabolic Stone Clinic, Kidney Disease Program, University of Louisville School of Medicine; Consulting Staff, Louisville Veterans Affairs Hospital

16. Clin Toxicol (Phila). 2011 Apr;49(4):277-83. doi: 10.3109/15563650.2011.582471. High-dose insulin therapy in beta-blocker and calcium channel-blocker poisoning. Engebretsen KM1, Kaczmarek KM, Morgan J, Holger JS.

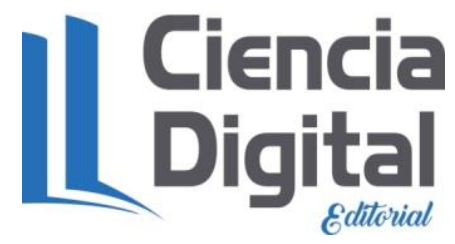




\section{Para citar el artículo indexado.}

Chacón N., Calderón A. \& Haro C. (2018). Síndrome de brash: bradicardia, falla renal, bloqueo auriculoventricular, shock e hiperkalemia. reporte de un caso. Revista electrónica Ciencia Digital 2(2), 578-589.. Recuperado desde:

http://cienciadigital.org/revistacienciadigital2/index.php/CienciaDigital/article/view/120/11 $\underline{1}$

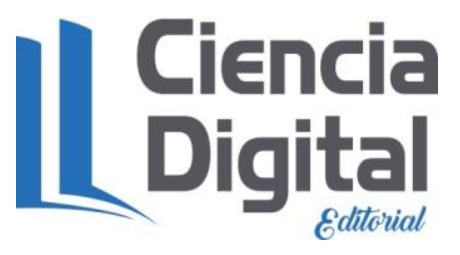

El artículo que se publica es de exclusiva responsabilidad de los autores y no necesariamente reflejan el pensamiento de la Revista Ciencia Digital.

El articulo queda en propiedad de la revista y, por tanto, su publicación parcial y/o total en otro medio tiene que ser autorizado por el director de la Revista Ciencia Digital.
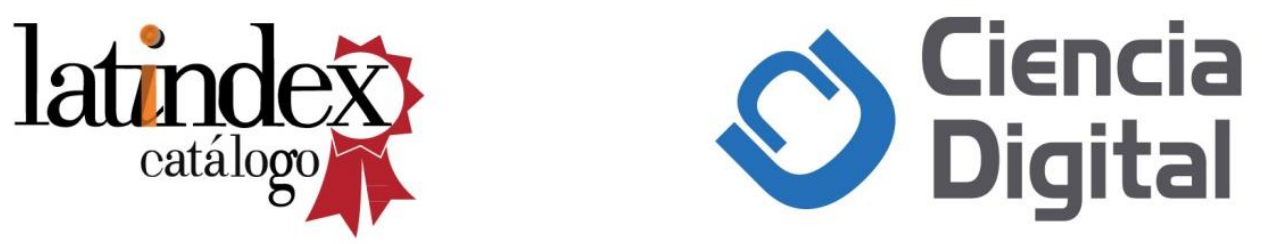